\title{
Splines and Wavelets: New Perspectives for Pattern Recognition
}

\author{
Michael Unser \\ Biomedical Imaging Group, \\ Swiss Federal Institute of Technology Lausanne (EPFL), \\ CH-1015 Lausanne Switzerland \\ Michael.Unser@epfl.ch
}

\begin{abstract}
We provide an overview of spline and wavelet techniques with an emphasis on applications in pattern recognition. The presentation is divided in three parts. In the first one, we argue that the spline representation is ideally suited for all processing tasks that require a continuous model of the underlying signals or images. We show that most forms of spline fitting (interpolation, least-squares approximation, smoothing splines) can be performed most efficiently using recursive digital filtering. We also discuss the connection between splines and Shannon's sampling theory. In the second part, we illustrate their use in pattern recognition with the help of a few examples: highquality interpolation of medical images, computation of image differentials for feature extraction, B-spline snakes, image registration, and estimation of optical flow. In the third and last part, we discuss the fundamental role of splines in wavelet theory. After a brief review of some key wavelet concepts, we show that every wavelet can be expressed as a convolution product between a Bspline and a distribution. The B-spline constitutes the regular part of the wavelet and is entirely responsible for its key mathematical properties. We also describe fractional B-spline wavelet bases, which have the unique property of being continuously adjustable. As the order of the spline increases, these wavelets converge to modulated Gaussians which are optimally localized in time (or space) and frequency.
\end{abstract}

\section{Splines and Continuous/Discrete Signal Processing}

What follows is a brief synopsis of the presentation, with some pointers to the relevant literature.

Splines provide a unifying framework for linking the continuous and discrete domains. They are well understood theoretically, and are ideally suited for performing numerical computations [6]. This makes them the perfect tool for solving a whole variety of signal and image processing (or pattern recognition) problems that are best formulated in the continuous domain but call for a discrete solution [21]. This leads to a class of computational techniques that we refer to as "continuous/discrete signal processing", and which may be best summarized by the motto "think analog, act digital".

The cardinal splines, which are the type of splines considering here, were invented by Schoenberg almost 60 years ago [14]. These polynomial splines are 1D functions that 
are defined on a uniform grid with knots at the integers when the degree is odd, or at the mid-integers when the degree is even. For each segment defined by two successive knots, the spline is a polynomial of degree $n$; the polynomial pieces are patched together at the knots in a way that guarantees the continuity of the function and of all its derivative up to order $(n-1)$. Schoenberg in his landmark paper showed that these splines could be represented most conveniently in terms of basis functions that are integer translates of a generating function: the B-spline of degree $n$. These $\mathrm{B}-$ splines have a number of very desirable properties. They have a simple analytical form (piecewise polynomial of degree $n$ ) that facilitates their manipulation [6, 23]. In our earlier work, we have shown that most B-spline computations can be made using digital filters [22, 23], provided that the grid is regular, which is always the case in image/signal processing.

The B-splines satisfy a two-scale relation which makes them prime candidates for constructing wavelet bases [24]. In this respect, B-splines form a category apart since the scaling relation holds for any positive integer $m$-and not just powers of two; this property can be used advantageously for designing fast multi-scale filtering algorithms [26] .

Splines have excellent approximation properties, mainly because the underlying Bspline basis functions are very regular $[19,4,3]$. They are also optimal in the sense that they provide the signal interpolant with the least oscillating energy [13]. Finally, the spline framework allows for a progressive transition between the two extreme signal representations: the piecewise-constant model (spline of degree zero) that uses the most localized, but least regular, basis functions, and the bandlimited model that corresponds to a spline of infinite degree [1]. Also note that there are recent extensions of Shannon's sampling theory that consider spline-like representations of functions instead of the traditional bandlimited model [20].

\section{Splines: Applications in Pattern Recognition}

The primary applications of splines in pattern recognition are sampling and interpolation, feature extraction, image matching, and motion analysis. In the presentation, we briefly discuss the following topics:

\subsection{High-Quality Image Interpolation}

When compared to other interpolation algorithms, splines provide the best tradeoff in terms of quality and of computational cost. In other words, if you want to improve interpolation quality, your best choice over any other method is to increase the order of the spline. This is a finding that has been confirmed independently by several research teams $[16,11,10,9]$.

\subsection{Feature Extraction}

After fitting the image with a spline, it is straightforward to compute exact image derivatives to derive gradients or Hessians for the detection of various image features such as contours or ridges [22] 


\subsection{Snakes and Active Contour Models}

The B-spline representation is also well suited for describing 2D curves [2]. These parametric curves can be optimized to detect object boundaries in images using snake-like algorithms $[12,5]$. An advantage of the spline model is that it gives a direct control on the smoothness of the curve.

\subsection{Image Registration}

There are various versions of spline-based algorithms for the registration of intramodal or inter-modal medical images [18, 17]. These can correct for rigid-body deformations with subpixel accuracy, even when the noise level is very high. Variants of these algorithms for elastic deformation are also available [7, 8]. Note that, in these latter works, both the image and the deformation function are represented by splines.

\subsection{Motion Analysis}

A good illustration of this topic is an optical flow algorithm that computes cardiac movement from ultrasound images of the heart [15]. This technique estimates the parameters of a local affine motion model over a sliding B-spline window. The method is implemented within a multi-scale framework using a wavelet-like algorithm for the efficient computation of weighted B-spline inner-products and moments.

\section{Fractional Splines and Wavelet Theory}

The polynomial splines have also been extended to fractional degrees [27]. The basic constituents of these fractional splines are piecewise power functions of degree $\alpha$. One constructs the corresponding B-splines through a localization process similar to the classical one, replacing finite differences by fractional differences. The fractional B-splines share virtually all the properties of the classical B-splines, including the two-scale relation. One of their key property is that these functions are closed under fractional differentiation; in other words, the fractional derivative of order $s$ (i.e., the multiplication by $(j \omega)^{s}$ in the frequency domain) of a spline of degree $\alpha$ yields a spline of degree $(\alpha-s)$.

We have shown recently that these fractional B-splines play a fundamental role in wavelet theory [28]. Their presence as a convolutional factor is necessary for the wavelet transform to be mathematically well-defined. More precisely, we can show that five key wavelet properties-vanishing moments, order of approximation, reproduction of polynomials, smoothness of the basis functions, multi-scale differentiation - are all to be attributed to the B-spline that lies hidden within. In other words, there cannot be wavelets without splines.

Fractional B-splines can also be used to construct new fractional wavelet basis functions that are unique in several respects. These basis functions are adjustable in a continuous manner. This gives the user a full control over all key wavelet properties: 
the parametric form of the basis functions, their smoothness, the order and multi-scale differentiability properties of the transform, and, finally, the number of vanishing moments. Interestingly, the spline degree $\alpha$ also controls the size (i.e., the spatial extent) of the basis functions. For instance, for the B-spline family, the basis functions (resp., the wavelets) converge to Gaussians (resp., modulated Gaussians or Gabor functions) with a standard deviation (or an equivalent window size) that is proportional to $\sqrt{\alpha+1}$. This also means that these functions, for $\alpha$ sufficiently large (say, $\alpha>2$ ), will tend to be optimally localized in the sense of the Heisenberg uncertainty principle; in other words, the product of their space and frequency uncertainties will tend to the minimum that is achievable. This result constitutes a fractional generalization of an earlier theorem for polynomial B-spline wavelets with integer order [25].

More information on splines and on fractional wavelets, including software $(\mathrm{C}$ and Java), demos, and papers, can be found at: http://bigwww.epfl.ch/

\section{References}

[1] A. Aldroubi, M. Unser and M. Eden, Cardinal spline filters: Stability and convergence to the ideal sinc interpolator, Signal Processing, 28 (1992), pp. 127-138.

[2] R. H. Bartels, J. C. Beatty and B. A. Barsky, Splines for use in computer graphics, Morgan Kaufmann, Los Altos, CA, 1987.

[3] T. Blu and M. Unser, Quantitative Fourier analysis of approximation techniques: Part II-wavelets, IEEE Transactions on Signal Processing, 47 (1999), pp. 2796-2806.

[4] T. Blu and M. Unser, Quantitative Fourier analysis of approximation techniques: Part Iinterpolators and projectors, IEEE Transactions on Signal Processing, 47 (1999), pp. 2783-2795.

[5] P. Brigger, J. Hoeg and M. Unser, B-spline snakes: a flexible tool for parametric contour detection, IEEE Transactions on Image Processing, 9 (2000), pp. 1484-1496.

[6] C. de Boor, A practical guide to splines, Springer-Verlag, New York, 1978.

[7] J. Kybic, P. Thévenaz, A. Nirkko and M. Unser, Unwarping of unidirectionally distorted EPI images, IEEE Transactions on Medical Imaging, 19 (2000), pp. 80-93.

[8] J. Kybic and M. Unser, Fast Parametric Elastic Image Registration, IEEE Transactions on Image Processing (in press).

[9] T. M. Lehmann, C. Gönner and K. Spitzer, Addendum: B-spline interpolation in medical image processing, IEEE Transactions on Medical Imaging, 20 (2001), pp. 660-665.

[10] T. M. Lehmann, C. Gönner and K. Spitzer, Survey: Interpolation methods in medical image processing, IEEE Transactions on Medical Imaging, 18 (1999), pp. 1049-1075.

[11] E. H. W. Meijering, W. J. Niessen and M. A. Viergever, Quantitative evaluation of convolution-based methods for medical image interpolation, Medical Image Analysis, 5 (2001), pp. 111-126.

[12] S. Menet, P. Saint-Marc and G. Medioni, B-snakes: implementation and application to stereo, Image Understanding Workshop, DARPA, 1990, pp. 720-726.

[13] P. M. Prenter, Splines and variational methods, Wiley, New York, 1975.

[14] I. J. Schoenberg, Contribution to the problem of approximation of equidistant data by analytic functions, Quart. Appl. Math., 4 (1946), pp. 45-99, 112-141.

[15] M. Sühling, M. Arigovindan, C. Jansen, P. Hunziker and M. Unser, Myocardial motion analysis and visualization from echocardiograms, SPIE Medical Imaging (MI'03), SPIE, San Diego, CA, 2003, pp. 306-313. 
[16] P. Thévenaz, T. Blu and M. Unser, Interpolation revisited, IEEE Transactions on Medical Imaging, 19 (2000), pp. 739-758.

[17] P. Thévenaz and M. Unser, Optimization of mutual information for multiresolution image registration, IEEE Transactions on Image Processing, 9 (2000), pp. 2083-2099.

[18] P. Thévenaz and M. Unser, A pyramid approach to sub-pixel image fusion based on mutual information, IEEE Int. Conf. on Image Processing, IEEE, Lausanne, Switzerland, 1996, pp. 265-268.

[19] M. Unser, Approximation power of biorthogonal wavelet expansions, IEEE Trans. Signal Processing, 44 (1996), pp. 519-527.

[20] M. Unser, Sampling-50 years after Shannon, Proceedings of the IEEE, 88 (2000), pp. $569-587$.

[21] M. Unser, Splines: A perfect fit for signal and image processing, IEEE Signal Processing Magazine, 16 (1999), pp. 22-38.

[22] M. Unser, A. Aldroubi and M. Eden, B-spline signal processing: Part II-efficient design and applications, IEEE Trans. Signal Processing, 41 (1993), pp. 834-848.

[23] M. Unser, A. Aldroubi and M. Eden, B-spline signal processing: Part I-theory, IEEE Trans. Signal Processing, 41 (1993), pp. 821-833.

[24] M. Unser, A. Aldroubi and M. Eden, A family of polynomial spline wavelet transforms, Signal Processing, 30 (1993), pp. 141-162.

[25] M. Unser, A. Aldroubi and M. Eden, On the asymptotic convergence of B-spline wavelets to Gabor functions, IEEE Trans. Information Theory, 38 (1992), pp. 864-872.

[26] M. Unser, A. Aldroubi and S. J. Schiff, Fast implementation of the continuous wavelet transform with integer scales, IEEE Trans. Signal Processing, 42 (1994), pp. 3519-3523.

[27] M. Unser and T. Blu, Fractional splines and wavelets, SIAM Review, 42 (2000), pp. 4367.

[28] M. Unser and T. Blu, Wavelet theory demystified, IEEE Transactions on Signal Processing, 51 (2003), pp. 470-483. 